\title{
Comparison of Growth and the Cytokines Induced by Pathogenic Yersinia enterocolitica Bio-Serotypes 3/0: 3 and 2/0: 9
}

\author{
Haoshu Yang ${ }^{1,2 \dagger}$, Wenpeng Gu ${ }^{3 \dagger}$, Haiyan Qiu ${ }^{1 \dagger}$, Guixiang Sun ${ }^{4}$, Junrong Liang ${ }^{1}$, Kewei $\mathrm{Li}^{1}$, \\ Yuchun Xiao ${ }^{1}$, Ran Duan ${ }^{1}$, Huaiqi Jing ${ }^{1 *}$ and Xin Wang ${ }^{1 *}$ \\ ${ }^{1}$ National Institute for Communicable Disease Control and Prevention, Chinese Center for Disease Control and Prevention, \\ State Key Laboratory for Infectious Disease Prevention and Control, Collaborative Innovation Center for Diagnosis and \\ Treatment of Infectious Diseases, Beijing, China, ${ }^{2}$ Lianyungang Center of Disease Control and Prevention, Lianyungang, \\ China, ${ }^{3}$ Yunnan Provincial Centres for Disease Control and Prevention, Kunming, China, ${ }^{4}$ Department of Public Health, \\ Xuzhou Medical College, Xuzhou, China
}

Pathogenic Yersinia enterocolitica is widely distributed in China where the primary bio-serotypes are 3/0: 3 and 2/O: 9. Recently, the distribution of 2/O: 9 strains are being gradually replaced by 3/O: 3 strains where presently 3/O: 3 strains are the major

OPEN ACCESS

Edited by:

Dongsheng Zhou,

Beijing Institute of Microbiology and

Epidemiology, China

Reviewed by:

Xue-jie Yu,

University of Texas Medical Branch,

USA

Shiwen Wang,

National Institute for Viral Disease and

Prevention, China

*Correspondence:

Huaiqi Jing

jinghuaiqi@icdc.cn

Xin Wang

wangxin@icdc.cn

${ }^{\dagger}$ These authors have contributed equally to this work.

Received: 20 February 2017 Accepted: 12 April 2017 Published: 01 May 2017

Citation:

Yang H, Gu W, Qiu H, Sun G, Liang J,

Li K, Xiao Y, Duan R, Jing H and Wang $X$ (2017) Comparison of Growth and the Cytokines Induced by Pathogenic Yersinia enterocolitica Bio-Serotypes 3/O: 3 and 2/0: 9 .

Front. Cell. Infect. Microbiol. 7:158. doi: 10.3389/fcimb.2017.00158 pathogenic $Y$. enterocolitica in China. To identify the growth conditions and cytokines induced by $Y$. enterocolitica and providing some clues for this shift, we performed competitive growth in vitro and in vivo for these two bio-serotype strains; and we also compared the cytokines induced by them in infected BALB/C mice. We found 2/O: 9 strains grew more in vitro, while 3/O: 3 strains grew more in vivo regardless of using single cultures or mixed cultures. The cytokines induced by the two strains were similar: interleukin-6 (IL-6), IL-9, IL-13, granulocyte colony-stimulating factor (G-CSF), chemokines $(\mathrm{KC})$, monocyte chemotactic protein 1 (MCP-1), macrophage inflammation protein-1 $\alpha(\mathrm{MIP}-1 \alpha)$, tumor necrosis factor- $\alpha(\mathrm{TNF}-\alpha)$, and RANTES were statistically up-regulated upon activation of normal T cells compared to the control. The cytokine values were higher in mixed infections than in single infections except for IL-6, G-CSF, and $\mathrm{KC}$. The data illustrated the different growth of pathogenic $Y$. enterocolitica bio-serotype 3/O: 3 and 2/O: 9 in vitro and in vivo, and the cytokine changes induced by the two strains in infected BALB/C mice. The growth comparisons of two strains maybe reflect the higher pathogenic ability or resistance to host immune response for $Y$. enterocolitica bio-serotype 3/O: 3 and maybe it as one of the reason for bacteria shift.

Keywords: Yersinia enterocolitica, competitive inhibition, cytokines, bio-serotypes 3/O: 3, bio-serotypes 2/0: 9

\section{INTRODUCTION}

Yersinia enterocolitica is widely distributed in the natural world; and being one of the few intestinal bacteria that can grow at low temperatures, the animal hosts of $Y$. enterocolitica are widely distributed, including livestock, poultry, rodents, reptiles, and aquatic animals. It causes gastrointestinal symptoms and systemic disease, e.g., liver and spleen abscess (Thoerner et al., 2003), reactive arthritis, erythema nodosum, and anemic goiter (Heyma et al., 1986; Stuart and Woodward, 1992; Gaede and Heesemann, 1995; Wang et al., 2012); and can cause septicemia 
and death (Bottone, 1999). Biotyping and serotyping are common and valuable methods for Y. enterocolitica identification where at present $Y$. enterocolitica is divided into six biotypes $(1 \mathrm{~A}, 1 \mathrm{~B}, 2,3,4$, and 5$)$ and 60 serotypes. The highly pathogenic $Y$. enterocolitica bioserotype 1B/O:8 strain was distributed worldwide (Wang et al., 2008, 2010, 2012; Liang et al., 2012); while the other bio-serotypes were the lower or non-pathogenic strains and distributed all over the world (Wang et al., 2011). From our previous investigation, 58 serotypes of $Y$. enterocolitica had been found and no highly 1B/O: 8 strain has been isolated in China (Wang et al., 2008; Gu et al., 2012). The bio-serotype 3/O: 3 and 2/O: 9 strains were the primary types found in China.

Since the 1990s, the isolation numbers of bio-serotype 2/O: 9 strains have decreased, while the 3/O: 3 strains became the dominant bio-serotype in China. From 2009 to 2011 in a national survey for Y. enterocolitica, 2/O: 9 almost disappeared where only six O: 9 strains were identified from 862 Y. enterocolitica (Liang et al., 2012). The mechanisms of this change were not known, and therefore to determine the reason, we performed comparative growth of the two strains in vitro and in vivo, and compared the bacteria produced cytokine changes of the two strains in infected $\mathrm{BALB} / \mathrm{C}$ mice.

\section{MATERIALS AND METHODS}

\section{Bacterial Strains}

NX1998-SA98-837 (bio-serotype 3/O: 3, strain A) and NX1998SA98-835 (bio-serotype 2/O: 9, strain B) were selected; both strains were isolated from Ningxia Province in 1998. We chose additional two pathogenic strains BJ2009-3-248 (3/O: 3, strain C) and BJ2009-3-247 (2/O: 9, strain D) isolated from Beijing in 2009. All four strains carried ail, ystA, virF, and yadA virulence genes, indicated that the strains were pathogenic ones.

\section{In vitro Cultures}

Two colonies of strain A and B were selected either as a single culture, or the strains were mixed as mixed cultures. All the cultures were inoculated in BHI broth and incubated at $25^{\circ} \mathrm{C}$ for $48 \mathrm{~h}$, and shaken at $120 \mathrm{rpm}$. Colony counts were performed every $3 \mathrm{~h}$, and each colony in the mixed bacterial culture was identified using monoclonal antibodies grown in our laboratory; and finally comparative growth curves were drawn. The experimental procedure was performed twice for each strain; and strains $\mathrm{C}$ and $\mathrm{D}$ were also included.

\section{In vivo Cultures}

Six week-old healthy female BALB/C mice were purchased from the Chinese Academy of Food and Drug Testing Laboratory Animal Resource Center. Experiments were performed using the four strains as mentioned above. For each experiment, O: 3 and O: 9 strains were mixed to infect animals using equal number of each bacterium in the experimental groups; O: 3 and O: 9 strains singly infected mice were the two control groups (O: 3 control group and O: 9 control group), and healthy mice were selected as a blank group. Approximately $10^{7} \mathrm{cfu} / \mathrm{ml}$ of the bacteria were used for intraperitoneal injection, three mice were removed randomly at $3,6,9,12,15,18,21,24,27,30,33,36,39,42,45$, and $48 \mathrm{~h}$ after infection and sub-ocular sinus blood was separated and the serum was stored at $-70^{\circ} \mathrm{C}$. Mice were sacrificed by cervical dislocation; the spleens were removed after immersion disinfection. Three spleens were mixed with $3 \mathrm{ml}$ PBS to form a suspension, and diluted 10 -fold to $10^{-3}$; and the appropriate concentration to coat plates at $3 \mathrm{~h}$ was made. The plates were cultured at $25^{\circ} \mathrm{C}$ for $24 \mathrm{~h}$.

\section{Cytokines}

The content of cytokines from each group of mice was measured and the serum levels of IL- $1 \alpha$, IL-1 $\beta$, IL-2, IL-3, IL-4, IL-5, IL-6, IL-9, IL-10, IL-12p40, IL-12p70, IL-13, IL-17 $\alpha$, eotaxin, G-CSF, GM-CSF, IFN-r, KC, MCP - 1 , MIP- $1 \alpha$, MIP- $1 \beta$, and RANTES were determined using a Bio-Plex mouse 23 cytokine kit purchased from the BioRad Company (USA). TNF- $\alpha$ was measured using an ELISA kit purchased from the R\&D Company (USA). After processing the data, a comparison of the cytokine levels in the three groups of mice was performed.

\section{Statistical Analysis}

The amount of bacteria at each time point was bacterial counts per milliliter and each cytokine measured value were expressed as mean \pm standard deviation. The statistical differences between each group were analyzed using analysis of variance or the $T$-test where differences had significance when $P \leq 0.05$.

\section{Ethics Statement}

The animals were handled according to the national criterion for animal investigation of China (Ethics Review Committee [Institutional Review Board (IRB)] of National Institute for Communicable Disease Control and Prevention, Chinese Center for Disease Control and Prevention). The experimental and detection protocols were carried out in accordance with relevant guidelines and regulations. The ethics of this study was approved by Ethics Review Committee of National Institute for Communicable Disease Control and Prevention, Chinese Center for Disease Control and Prevention. The License numbers were ICDC-2015001 and ICDC-2015002.

\section{RESULTS}

The initial bacteria concentration of NX1998-SA98-837 was 1.03 $\times 10^{8} \mathrm{cfu} / \mathrm{ml}$ and NX1998-SA98-835 was $0.89 \times 10^{8} \mathrm{cfu} / \mathrm{ml}$; and BJ2009-3-248 was $0.65 \times 10^{8} \mathrm{cfu} / \mathrm{ml}$ and BJ2009-3-247 was 0.87 $\times 10^{8} \mathrm{cfu} / \mathrm{ml}$.

The growth of different bio-serotype strains in vitro or in vivo was different. Comparing the growth curves of the four strains in vitro, we found that 2/O: 9 strains had statistically significant higher growth than 3/O: 3 strains (Figures 1A,B), either in single culture or mixed cultures. However, the growth curves of four strains in vivo were inverse, 3/O: 3 grew statistically significantly higher than the 2/O: 9 (Figures 1C,D), either in single infection or mixed infection.

The cytokine changes in mouse sera infected with the two bio-serotype strains were similar where only IL-6, IL-8, IL-13, GCSF, KC, MCP-1, MIP-1 $\alpha$, RANTES, and TNF- $\alpha$ were different statistically compared to the control group (Figure 2). For IL-8, 
A

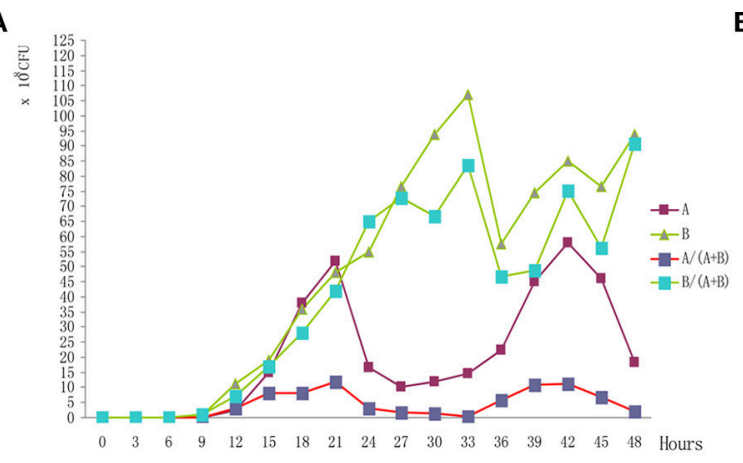

C

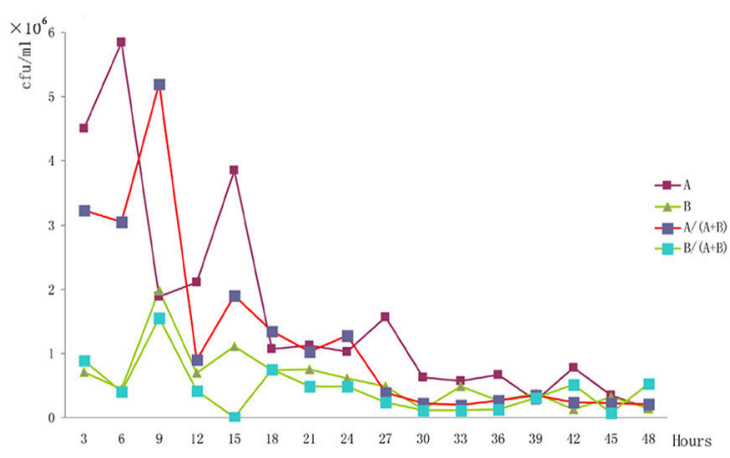

B

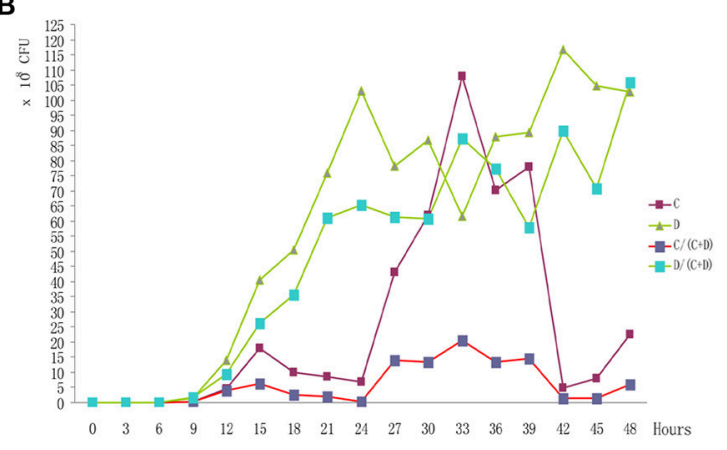

D

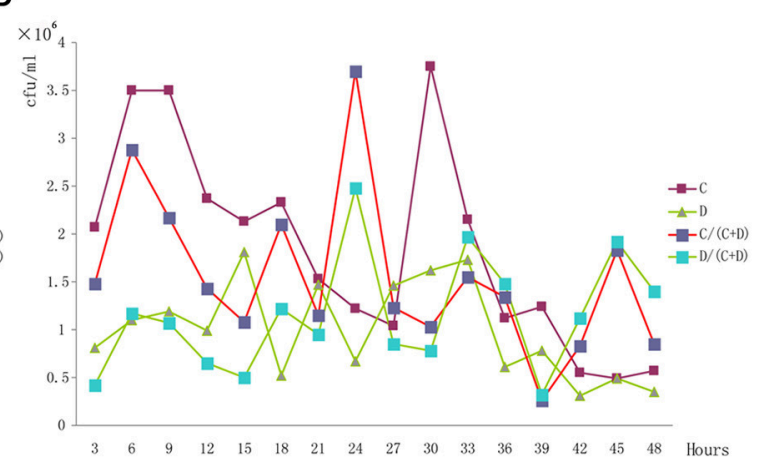

FIGURE 1 | Bacterial numbers of each strain in vitro and in vivo. (A) The growth curves of Ningxia strains in vitro. (B) The growth curves of Beijing strains in vitro. (C) The growth curves of Ningxia strains in vivo. (D) The growth curves of Beijing strains in vivo.

IL-13, MCP-1, MIP-1 $\alpha$, RANTES, and TNF- $\alpha$, the cytokine values using the mixed infection with two strains were higher than single infections; and there was no difference with $3 / \mathrm{O}: 3$ or $2 / \mathrm{O}: 9$ independently infected. For IL- 6 and $\mathrm{KC}$, the values from $3 / \mathrm{O}$ : 3 infections were higher than the 2/O: 9 infections and mixed infections; and for G-CSF, the values of 2/O: 9 infections were higher than 3/O: 3 and mixed infections. The experiments were repeated twice, and the all the results were consist.

\section{DISCUSSION}

In our study, the competitive growth of the two bio-serotype strains were totally different, the $3 / \mathrm{O}: 3$ grew greater than $2 / \mathrm{O}$ : 9 in vivo; conversely, the 2/O: 9 grew greater than 3/O: 3 in vitro. This suggested the 2/O: 9 grew better without a host; however, the 3/O: 3 had stronger resistance to the defense mechanisms of the hosts. Therefore, the clearance of the 3/O: 3 was slower than the 2/O: 9 in BALB/C mice; and might reflected the different pathogenic abilities. This showed the higher resistant ability of 3/O: 3 to host clearance than the 2/O: 9 , and might be a possible explanation for distribution of the two strains in human hosts.

Cytokines participate in cellular immunity, humoral immunity, hematogenesis regulation, cell proliferation, and injury repair; however, excess cytokine secretion leads to immunopathogenic effects. Some research showed excess cytokines induced host death after infections (Hancock et al.,
1986; Segura et al., 1999; Smith et al., 2008). Currently, the studies on different serotypes of $Y$. enterocolitica that cause different secretions of cytokines were shown (McNally et al., 2006). In our study here, the Yersinia highly susceptible BALB/C mice were used instead of cells (Autenrieth and Heesemann, 1992). Cytokines involved in this study, IL-6, IL-8, MCP-1, MIP- $1 \alpha$, and RANTES in a relatively short period of time reach their peaks in both the two control groups and the experimental group. These cytokines were inflammatory cytokines. This was consistent with the reported literature: Yersinia infection of epithelium (HeLa) cells can significantly enhance the levels of transcription and secretion of inflammatory cytokines MCP-1, GM-CSF, and TNF- $\alpha$. One hour after infection transcript levels of these cytokines began to increase and within 3-4 h reached their peak and then gradually decline (Kampik et al., 2000). These cytokines played important roles in the hosts' immune response against $Y$. enterocolitica and the cytokine changes for $Y$. enterocolitica infection were similar using cell infected models or animal infected models.

Previous studies showed the cytokine values of highly pathogenic $Y$. enterocolitica were higher than the lower pathogenic strains (Gu et al., 2013). Brucella and 2/O: 9 bioserotype $Y$. enterocolitica caused different cytokine changes, involving their different pathogenic ability and life cycles (Wang et al., 2013). However, in our study lower pathogenic $Y$. enterocolitica had similar cytokine changes compared with 3/O: 3 


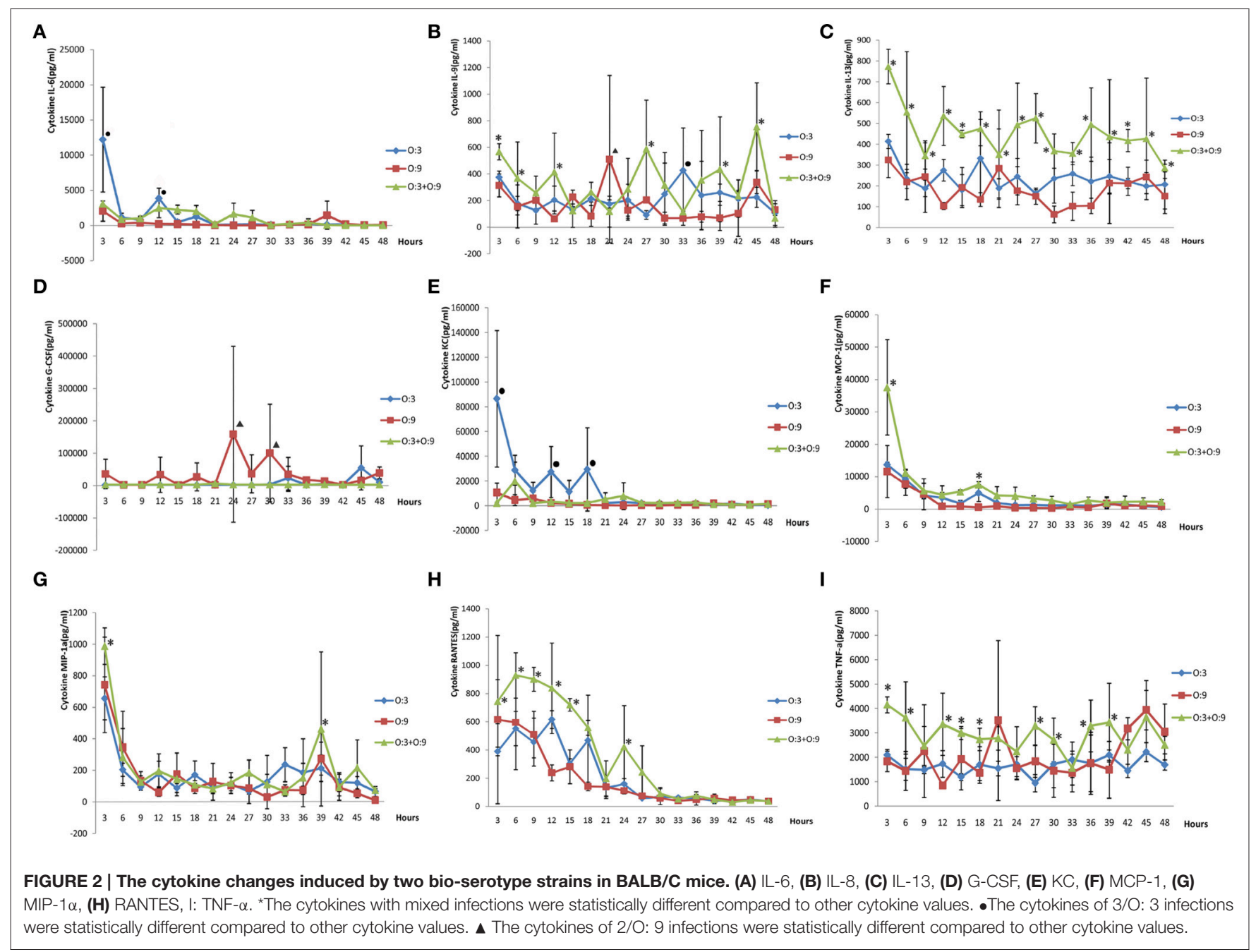

and 2/O: 9 strains. Therefore, the similar cytokine changes were caused by analogical pathogenic ability of the Y. enterocolitica strains. The different pathogenic abilities or life cycles were important for cytokine changes in $Y$. enterocolitica infections. The cytokines induced by two kinds of strains were similar, while the growth conditions were different; maybe this reflected the different pathogenic ability or defense ability to hosts, and it may as one of the reason for bacteria shift.

\section{AUTHOR CONTRIBUTIONS}

Design of the work: HJ and XW. Do the experiments: HY, HQ, JL, KL, YX, and RD. Analysis the data: HY and WG. Drafting

\section{REFERENCES}

Autenrieth, I. B., and Heesemann, J. (1992). In vivo neutralization of tumor necrosis factor-alpha and interferon-gamma abrogates resistance to Yersinia enterocolitica infection in mice. Med. Microbiol. Immunol. 181, 333-338. doi: 10.1007/BF00191545 the work: WG. Revising it critically for important intellectual content: GS.

\section{FUNDING}

This work was supported by the National Sci-Tech Key Project (2012ZX10004-201, 2012ZX10004-212, and 2013ZX100 04203-002).

\section{ACKNOWLEDGMENTS}

We thank Liuying Tang and Jim Nelson for critical reading of and helpful comments on our manuscript.

Bottone, E. J. (1999). Yersinia enterocolitica: overview and epidemiologic correlates. Microbes Infect. 1, 323-333. doi: 10.1016/S1286-4579(99)80028-8

Gaede, K. I., and Heesemann, J. (1995). Arthritogenicity of genetically manipulated Yersinia enterocolitica serotype O8 for Lewis rats. Infect. Immun. 63, 714-719.

Gu, W., Wang, X., Qiu, H., Cui, B., Zhao, S., Zheng, H., et al. (2013). Comparison of cytokine immune responses to Brucella abortus and Yersinia enterocolitica 
serotype O:9 infections in BALB/c mice. Infect. Immun. 81, 4392-4398. doi: 10.1128/IAI.00856-13

Gu, W., Wang, X., Qiu, H., Luo, X., Xiao, D., Xiao, Y., et al. (2012). Comparative antigenic proteins and proteomics of pathogenic Yersinia enterocolitica bioserotypes 1B/O: 8 and 2/O: 9 cultured at 25 degrees $\mathrm{C}$ and 37 degrees C. Microbiol. Immunol. 56, 583-594. doi: 10.1111/j.1348-0421.2012.00478.x

Hancock, G. E., Schaedler, R. W., and MacDonald, T. T. (1986). Yersinia enterocolitica infection in resistant and susceptible strains of mice. Infect. Immun. 53, 26-31.

Heyma, P., Harrison, L. C., and Robins-Browne, R. (1986). Thyrotrophin (TSH) binding sites on Yersinia enterocolitica recognized by immunoglobulins from humans with Graves' disease. Clin. Exp. Immunol. 64, 249-254.

Kampik, D., Schulte, R., and Autenrieth, I. B. (2000). Yersinia enterocolitica invasin protein triggers differential production of interleukin-1, interleukin-8, monocyte chemoattractant protein 1, granulocyte-macrophage colonystimulating factor, and tumor necrosis factor alpha in epithelial cells: implications for understanding the early cytokine network in Yersinia infections. Infect. Immun. 68, 2484-2492. doi: 10.1128/IAI.68.5.24842492.2000

Liang, J., Wang, X., Xiao, Y., Cui, Z., Xia, S., Hao, Q., et al. (2012). Prevalence of Yersinia enterocolitica in pigs slaughtered in Chinese abattoirs. Appl. Environ. Microbiol. 78, 2949-2956. doi: 10.1128/AEM.07893-11

McNally, A., Dalton, T., La Ragione, R. M., Stapleton, K., Manning, G., and Newell, D. G. (2006). Yersinia enterocolitica isolates of differing biotypes from humans and animals are adherent, invasive and persist in macrophages, but differ in cytokine secretion profiles in vitro. J. Med. Microbiol. 55(Pt 12), 1725-1734. doi: 10.1099/jmm.0.46726-0

Segura, M., Stankova, J., and Gottschalk, M. (1999). Heat-killed Streptococcus suis capsular type 2 strains stimulate tumor necrosis factor alpha and interleukin-6 production by murine macrophages. Infect. Immun. 67, 4646-4654.

Smith, M. A., Takeuchi, K., Anderson, G., Ware, G. O., McClure, H. M., Raybourne, R. B., et al. (2008). Dose-response model for Listeria monocytogenes-induced stillbirths in nonhuman primates. Infect. Immun. 76, 726-731. doi: 10.1128/IAI.01366-06

Stuart, P. M., and Woodward, J. G. (1992). Yersinia enterocolitica produces superantigenic activity. J. Immunol. 148, 225-233.
Thoerner, P., Bin Kingombe, C. I., Bögli-Stuber, K., Bissig-Choisat, B., Wassenaar, T. M., Frey, J., et al. (2003). PCR detection of virulence genes in Yersinia enterocolitica and Yersinia pseudotuberculosis and investigation of virulence gene distribution. Appl. Environ. Microbiol. 69, 1810-1816. doi: 10.1128/AEM.69.3.1810-1816.2003

Wang, X., Cui, Z., Wang, H., Tang, L., Yang, J., Gu, L., et al. (2010). Pathogenic strains of Yersinia enterocolitica isolated from domestic dogs (Canis familiaris) belonging to farmers are of the same subtype as pathogenic $Y$. enterocolitica strains isolated from humans and may be a source of human infection in Jiangsu Province, China. J. Clin. Microbiol. 48, 1604-1610. doi: 10.1128/JCM.01789-09

Wang, X., Gu, W., Cui, Z., Luo, L., Cheng, P., Xiao, Y., et al. (2012). Multiple-locus variable-number tandem-repeat analysis of pathogenic Yersinia enterocolitica in China. PLoS ONE 7:e37309. doi: 10.1371/journal.pone.0037309

Wang, X., Gu, W., Qiu, H., Xia, S., Zheng, H., Xiao, Y., et al. (2013). Comparison of the cytokine immune response to pathogenic Yersinia enterocolitica bioserotype 1B/O:8 and 2/O:9 in susceptible BALB/C and resistant C57BL/6 mice. Mol. Immunol. 55, 365-371. doi: 10.1016/j.molimm.2013.03.017

Wang, X., Li, Y., Jing, H., Ren, Y., Zhou, Z., Wang, S., Kan, B., et al. (2011) Complete genome sequence of a Yersinia enterocolitica "Old World" (3/O:9) strain and comparison with the "New World" (1B/O:8) strain. J. Clin. Microbiol. 49, 1251-1259. doi: 10.1128/JCM.01921-10

Wang, X., Qiu, H., Jin, D., Cui, Z., Kan, B., Xiao, Y., et al. (2008). O:8 serotype Yersinia enterocolitica strains in China. Int. J. Food Microbiol. 125, 259-266. doi: 10.1016/j.ijfoodmicro.2008.04.016

Conflict of Interest Statement: The authors declare that the research was conducted in the absence of any commercial or financial relationships that could be construed as a potential conflict of interest.

Copyright (c) 2017 Yang, Gu, Qiu, Sun, Liang, Li, Xiao, Duan, Jing and Wang. This is an open-access article distributed under the terms of the Creative Commons Attribution License (CC BY). The use, distribution or reproduction in other forums is permitted, provided the original author(s) or licensor are credited and that the original publication in this journal is cited, in accordance with accepted academic practice. No use, distribution or reproduction is permitted which does not comply with these terms. 